


\title{
One Stage Laparoscopic Band Removal and Sleeve Gastrectomy after Failure of Gastric Banding in Morbidly Obese Patients
}

\author{
Mohab G. Elbarbary, MD; Youhanna Shohdy Shafik, MD, MRCS; Mahmoud Abdelbaky \\ Mahmoud, MD; Abd-Allah H. Ibrahim, MD \\ Department of General Surgery, Ain Shams University, Egypt
}

\begin{abstract}
Background: Obesity is one of the public health problems nowadays which is associated with a variety of diseases including the whole systems, specially the cardiovascular system. Bariatric surgeries believed to be the most effective long-term management for morbid obesity that decrease both morbidity and mortality. One of these surgeries is laparoscopic adjustable gastric banding.
\end{abstract}

Objective: To study the safety and effectiveness of single stage revision of failed gastric banding to sleeve gastrectomy only in uncomplicated cases who had failure of weight loss or regain weight after a period from having gastric banding.

Patients and methods: The present study is a retrograde observational study including 150 morbidly obese patients who previously had laparoscopic adjustable gastric banding and failed to lose adequate weight or regained weight. Patients were recruited and treated at Ain Shams university hospitals from December 2015 to December 2017. All patients had laparoscopic band removal and sleeve gastrectomy in the same procedure. All patients received information about surgical technique, risks of the operation and other options for treatment.

Results: The mean age of the cohort was $36 \pm 10$ years (range 20-60). Before band insertion the mean BMI of the cohort was $43.4 \pm 5.2$ (range 31.6-55.8). The mean interval between primary and revision surgery was $5.5 \pm 1$ year (range 2.8-9.6). The mean BMI of the study prior to revisional laparoscopic band removal and sleeve gastrectomy was $42 \pm 5.5$ (range 35-50). The excess body weight loss percentage (EBWL\%) in the first 3 months was $32 \%$ and $65 \%$ after 6 months.

Conclusion: Laparoscopic gastric band removal with conversion to sleeve gastrectomy in one operation is applicable for patients with failure of weight loss or regaining weight after adjustable gastric band. It can be done with low incidence of complications, and yet a weight loss results that is similar to primary sleeve gastrectomy.

Key words: Laparoscopic adjustable gastric banding, laparoscopic sleeve gastrectomy, body mass index.

\section{Introduction}

Obesity is one of the famous public health problems nowadays which is associated with variable diseases affecting the different systems of the body specially the cardiovascular system. It is obvious that diet and exercise failed to be a definitive treatment in $90 \%$ of obese patients. On the contrary, different bariatric surgeries showed an effective long-term outcome and marked improvement in the patients' quality of life.

Bariatric surgeries are considered the best treatment with long term results for morbid obesity that effectively decrease morbidity and mortality. Roux en-Y gastric bypass is considered one of the first operations which has been performed for decades. However, Because of its multiple long-term complications, a lot of restrictive only procedures, as laparoscopic adjustable gastric banding, had been developed and found to have lower morbidity and mortality. Although the long-term outcomes after the gastric banding procedure was not accepted as well but it's still safer than Roux en $-Y$ gastric bypass. $^{2}$

Despite the initial good results of both laparoscopic gastric banding (LAGB) and vertical banded gastroplasty (VBG) in the late 1990s that reached 54-58\% weight loss. However, the further followup proved that their high failure rates had reached (20-56\%) nowadays. ${ }^{3}$

The failures are commonly due to failure to lose weight or regaining weight after few years but other causes as band erosion, tube leakage, or port site troubles might be included. ${ }^{3}$ Laparoscopic sleeve gastrectomy (LSG) was performed for the first time in 1998 as a part of biliopancreatic diversion with duodenal switch (BPD-DS). It is believed that LSG used to reduce weight through reducing the 
size of the stomach and decreasing the ghrelin hormone produced by removal of orexigenic cells in the fundus of the stomach as well as accelerating gastric emptying. ${ }^{4}$

So, LSG used firstly as a first step followed by another operation which used to be Roux en-Y gastric bypass or biliopancreatic diversion and duodenal switch for the super-obese as it is proven to give better weight loss results and resolution of co-morbidities with lower rates of complications. Nowadays, LSG has become popular as a single stand-alone procedure with very good long-term results. ${ }^{5}$

For that reason, the laparoscopic sleeve gastrectomy has evolved as an effective restrictive bariatric surgery compared to the popular laparoscopic adjustable gastric banding. ${ }^{6}$

\section{Patients and methods}

This retrospective observational study was carried out at Ain Shams University hospitals. An informed consent was obtained from the patients for participation in our study. An approval by the Ethical Committee of the Faculty of Medicine, Ain Shams University was taken for the assessment.

Study design: The present study is an observational study including 100 morbidly obese patients who previously had laparoscopic adjustable gastric banding and failed to lose adequate weight or regained weight. Patients were recruited and treated at Ain Shams university hospitals from December 2015 to December 2017. All patients had laparoscopic band removal and sleeve gastrectomy in same session. All patients received information about surgical technique and risks of the operation and other options for treatment.

Inclusion Criteria: Age ranging between 1860 years. Body mass index between 35 and 50 . Patients who had laparoscopic gastric banding with failure of adequate weight loss or regaining weight after $2.8-9.6$ years.

Exclusion Criteria: Age $<18$ or $>60$ years. Obese patients with major cardiac, respiratory, renal or hepatic problems that interfere with anesthesia or laparoscopy.

Patients with complicated band after insertion, as well as sweet eaters were excluded.

\section{Steps of the Study:}

Preoperative patients were evaluated by: Full history and examination. History for the body mass index at the time of gastric banding before and at the time of conversion to LSG.

Questionnaire for psychological assessment of the patient and eating behavior. Routine laboratory studies, pelvi-abdominal ultrasound, and upper GI endoscopy to exclude band erosion.

Surgery: All the patients had conversion of gastric banding to laparoscopic sleeve gastrectomy in the same sitting under general anesthesia and under complete aseptic conditions and along with operative time collection. (Figures 1-9).

\section{Steps of operation: Anesthesia:}

All patients had general anesthesia. We advised the patient to have liquid diet in the 24 hours before surgery and at least nothing by mouth (NPO) 6-8 hours before surgery.

Prophylactic antibiotic intravenously and low molecular weight heparin (Clexan) subcutaneously were given within 30 to 120 minutes before surgery or with the induction of anesthesia.

\section{Surgical Technique}

The patient lied supine in bed in a reverse Trendelenburg position with splitted legs and his arms abducted. Elastic and intermittent compressing stockings were applied. The surgeon was positioned between the patient's legs and the camera-man to the patient's right side.

The patient was secured to the operating table.

The abdomen was insufflated with visi-port. A five trocars technique was used.

The first 12-mm visiport trocar was placed in the left mid clavicular line $2 \mathrm{~cm}$ from the costal margin. One 5-mm trocar was placed just below the xiphoid process, another $5 \mathrm{~mm}$ port at anterior axillary line and one in the right midclavicular line. The camera port was $15 \mathrm{~mm}$ port as it was used also for stapling about $20 \mathrm{~cm}$ from xiphisternum in the midline or slightly to the patient's left. The liver was retracted by $5 \mathrm{~mm}$ instrument through the upper port after dissection of adhesions (Figure 1).

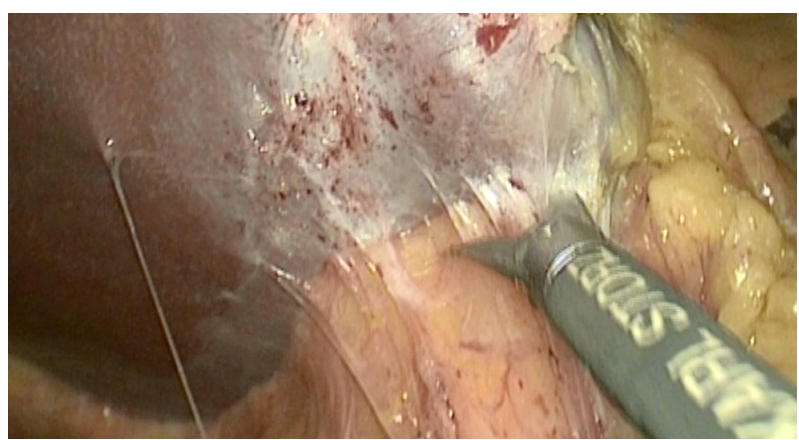

Fig 1: Liver retraction cutting any adhesions between it and the band. 
The band was freed of adhesions (mostly to the liver to allow liver retraction and gastroesophageal angle visualization) and the gastric wall exposed by cutting the gastrogastric tunnel over the band before removing the band.

Removal of the band capsule was very important to avoid postoperative leakage or stricture (Figures 2,3 ).

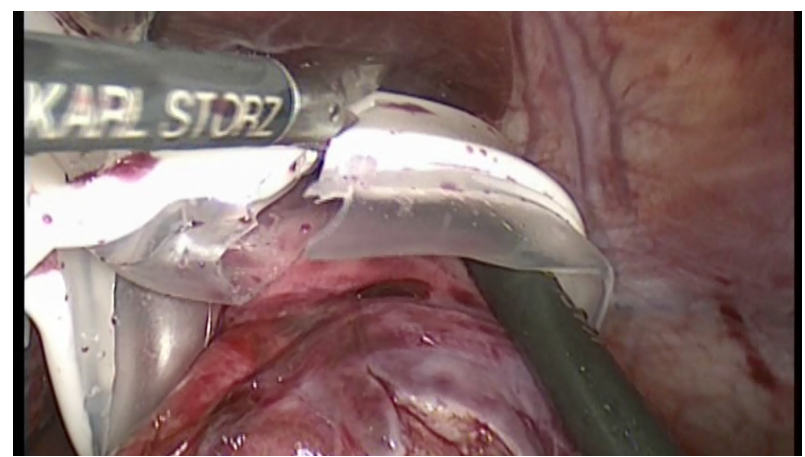

Fig 2: Cutting and removal of the band.

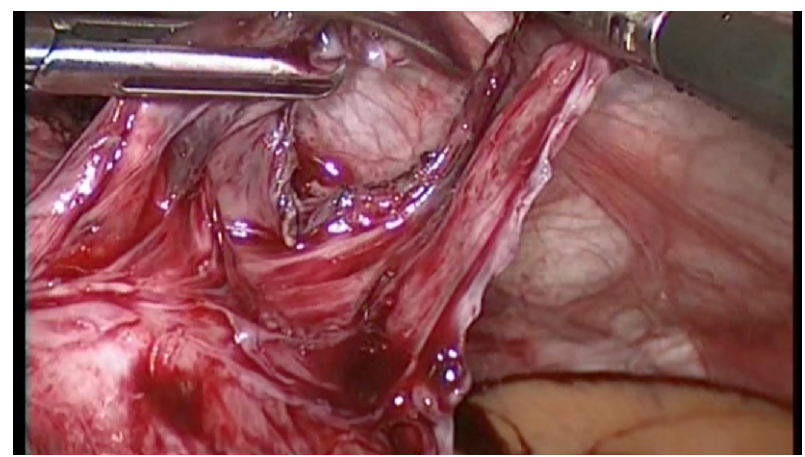

Fig 3: Dissection and division of the band capsule was very important to avoid postoperative stricture or leakage.

A one-stage strategy was followed (proceed directly to LSG). No buttressing was used. Dissection of greater curvature of the stomach from greater omentum using ligasure starting at the level of the crow's foot (Figure 4). Proximal dissection continued till complete visualization of the left crus and reaching angle of His (Figures $\mathbf{5 a}, \mathbf{5} \mathbf{b}$ ) and distal dissection till 2-3 cm away from the pylorus (Figure 6).

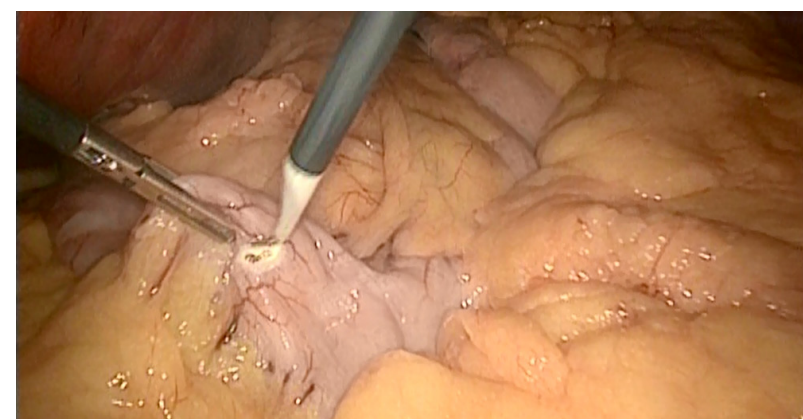

Fig 4: The mark done opposite craw foot to start greater omentum dissection.

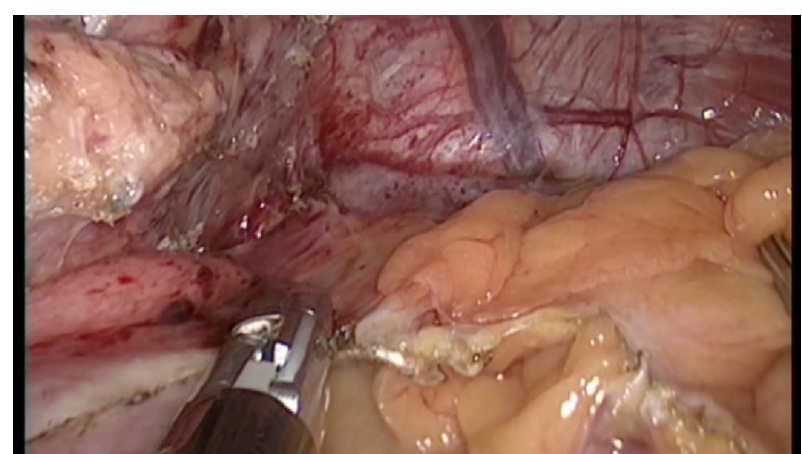

Fig 5a: Proximal dissection of the greater omentum till appearance of left crus of diaphragm.

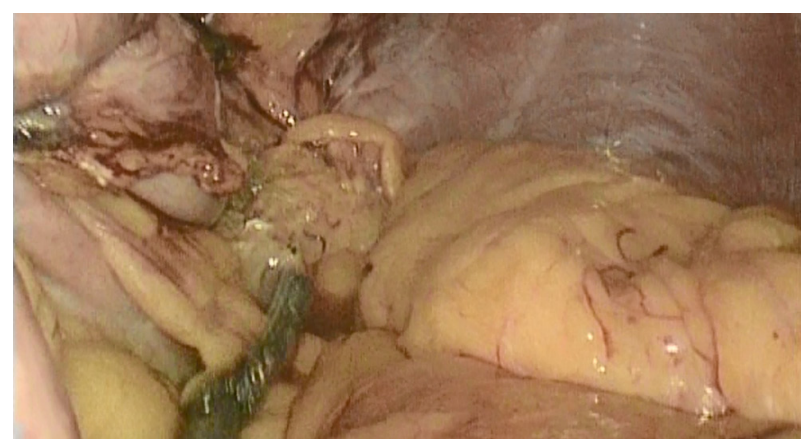

Fig 5b: Posterior dissection of greater omentum and the fundus to visulaize left crus.

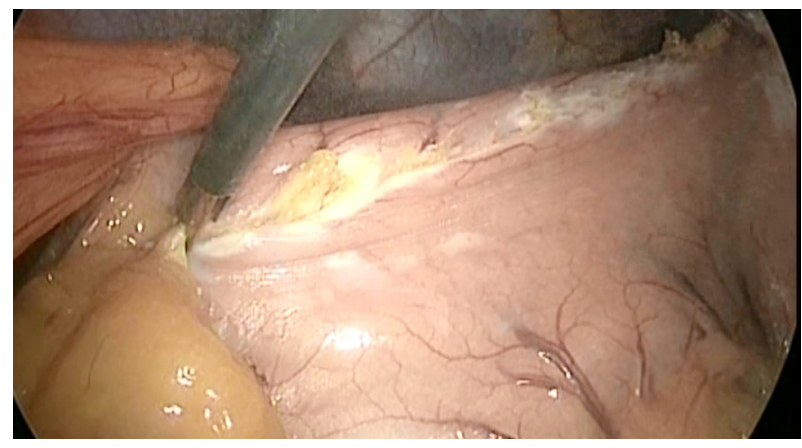

Fig 6: Distal dissetion of the greater omentum sparing $2-3 \mathrm{~cm}$ from pylorus.

The stomach was transected using an average six green cartridge guiding by a $36-\mathrm{F}$ orogastric tube that is pressed against the lesser curvature of the stomach (Figures 7-10).

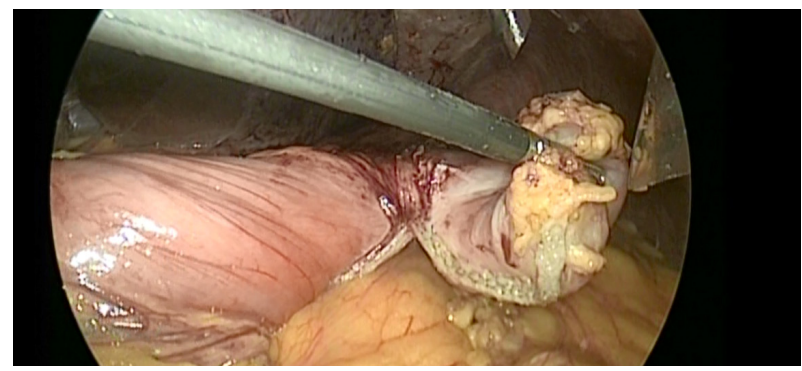

Fig 7: First stapler. 


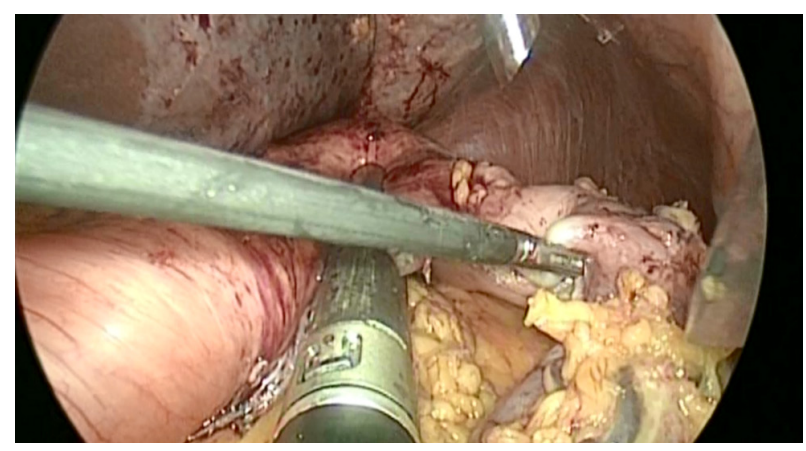

Fig 8: $3^{\text {rd }}$ stapler.

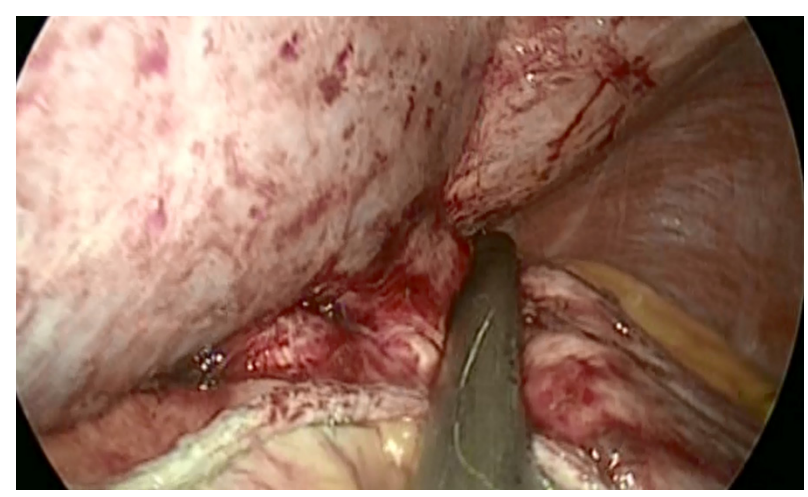

Fig 9: 4h $^{\text {th }}$ stapler.

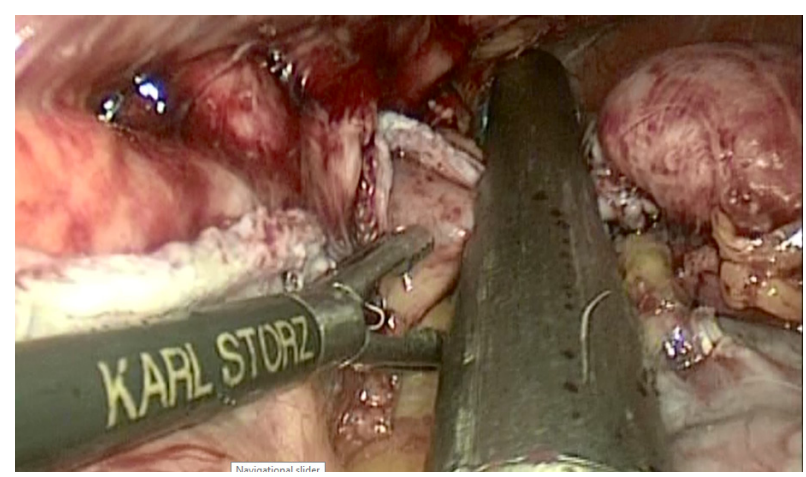

Fig 10: Last stapler.

Band was removed and the excluded part of the stomach was extracted from the abdomen through $15-\mathrm{mm}$ trocar site. This $15-\mathrm{mm}$ fascia lesion was closed by endoclose using absorbable suture. The orogastric tube was removed, $\mathrm{CO} 2$ released, and trocars removed under vision. Skin was closed subcuticularly after drain insertion through the $5 \mathrm{~mm}$ port to the patient left side.

Postoperative: We collected data including length of hospital stay, short-term complications as hemorrhage, leakage, hyperemesis, deep venous thrombosis and wound infection. Regular follow up of weight \& percent of excess weight loss (at 3, 6 months).

\section{Postoperative workup and follow up}

Usually, patients were discharged at the second postoperative day with liquid diet advised for 10 days. Oral crystal liquids were allowed at the second day of surgery. $30 \mathrm{ml}$ every hour in the early morning and if patient tolerating, clear fluids in the evening. Only clear fluids were allowed for 10 days. At the first or second postoperative day, the patient was discharged.

CT scan with oral diluted contrast was performed in patients with early signs of leakage suspicious (HR>120/min, febrile temperature and elevated CRP), but in case of high suspicion of leakage, a diagnostic laparoscopy was performed. Gastrografin study was performed in case of severe dysphagia or mild dysphagia lasting more than 3 months postoperatively. Bleeding from staple line or from port site diagnosed by $\mathrm{HR}>110$ and increasing along with drop in $\mathrm{Hb}>2 \mathrm{gm} / \mathrm{dl}$ was treated by blood transfusion in 10 patients.

Furthermore, elastic compression stockings (for $24 \mathrm{~h}$ postoperatively) were used, and subcutaneous low molecular weight heparin (LMWH) injection (daily dose of $40 \mathrm{U}$ ) was administered until discharge as a result of a relatively high rate of DVT and pulmonary embolism for those patients, LMWH was currently prescribed up until 1 week postoperatively. In addition, a proton pump inhibitor (omeprazole $40 \mathrm{mg} /$ day) was used for 6-8 weeks to prevent marginal ulcer formation.

Outpatient clinic visits were scheduled at 3 and 6 months postoperatively.

\section{Result \\ Statistical analysis}

The collected data was revised, coded, tabulated and introduced to a PC using statistical package for social science (SP 15.0.1 windows; SPSS Inc, Chicago, IL, 2001).

Data was presented and suitable analysis was done according to the type of data obtained for each parameter.

\section{Peri-operative outcomes}

The peri-operative characteristics of the patients presenting with weight regain (difference of BMI before and after band insertion and removal) and postoperative complications were included in Tables 1-4.

Table 1: Demographic and clinical data

\begin{tabular}{lcc}
\hline Age & Mean \pm SD & $36 \pm 10$ \\
"Years" & Range & $20-60$ \\
Sex & Male & $34(22.7 \%)$ \\
& Female & $116(77.3 \%)$ \\
\hline
\end{tabular}


Table 2: LAGB data

\begin{tabular}{lll}
\hline BMI before & Mean \pm SD & $43.4 \pm 5.2$ \\
"LAGB" & Range & $31.6-55.8$ \\
LAGB duration & Mean \pm SD & $5.5 \pm 1$ \\
"Years" & Range & $2.8-9.6$ \\
\hline
\end{tabular}

Table 3: Band removal and sleeve data

\begin{tabular}{lll}
\hline BMI before & Mean \pm SD & $42 \pm 5.5$ \\
"BR \& SL" & Range & $35-50$ \\
BR \& SL duration & Mean \pm SD & $2.24 \pm 0.2$ \\
"Years" & Range & $1.8-2.5$ \\
\hline
\end{tabular}

Table 4: Post-Operative Complications

\begin{tabular}{lc}
\hline Post-Operative Bleeding & $10(6.7 \%)$ \\
Post-Operative Leak & 0 \\
Blood Transfusion & $10(6.7 \%)$ \\
\hline
\end{tabular}

\section{Operative outcomes}

All patients had band removal and sleeve gastrectomy on one stage as there was no post laparoscopic banding complications. The reason for revisional surgery was failure to lose enough weight or regaining weight after a period from gastric banding (Table 5).

\section{Post-operative outcomes}

No major complications were noticed in the whole patients. There were ten patients with postoperative bleeding which was diagnosed by tachycardia and follow up CBC and treated conservatively by blood transfusion (2-3 units), with no incidence of leakage, DVT, portal vein thrombosis or any vitamin deficiency post-opertively (within first 6 months). There was no kind of intra-operative or postoperative sepsis recorded except for 15 patients who had infection at the site of band reservoir after removal and was treated by dressings and antibiotics. Mean hospital stay for the cohort was 2 days (range 2-4); with mean operative time $123 \pm 27$ minutes (range 75180) (Table 6).
Table 5: Operation related data

\begin{tabular}{lll}
\hline Operative time & Mean \pm SD & $123 \pm 27$ \\
"minutes" & Range & $75-180$ \\
Hospital stay & Mean \pm SD & $2 \pm 0$ \\
"days" & Range & $2-4$ \\
\hline
\end{tabular}

\section{Long-term outcomes}

At the follow-up period the mean BMI of the whole cohort had fallen to $36.8 \pm 3.8$ with a percentage of excess body weight loss of $32 \pm 4.7$ (range 8.4$43.6 \%$ ) in the first 3 months. And BMI drop to $34.3 \pm$ 3.1 with percentage of excess body weight loss of $65 \pm 5.7$ (range15.7-58.9\%) after 6 months.

Table 6: Post-Operative Outcome

\begin{tabular}{lll}
\hline \multirow{3}{3}{ months (BMI) } & Mean \pm SD & $36.8 \pm 3.8$ \\
& Range & $31.2-43.2$ \\
6 months (BMI) & Mean \pm SD & $34.3 \pm 3.1$ \\
& Range & $30-40$ \\
3 months \% EBWL & Mean \pm SD & $32 \pm 4.7$ \\
& Range & $8.4-43.6$ \\
6 months \% BEBWL & Mean \pm SD & $65 \pm 5.7$ \\
& Range & $15.7-58.9$ \\
\hline
\end{tabular}

\section{Discussion}

Buchwald and his colleagues ${ }^{7}$ showed that the spread of bariatric surgeries worldwide increase with the rise in the rate of global obesity.

One of the commonest bariatric surgeries in the world in the early 90 s was LAGB reaching $80 \%$ in the Asia and pacific. This was attributed to its safety and easy learning curve with minimal morbidity and almost no mortality results. However, because of its high rates of failure and complications LAGB was famous to be followed by a revisional surgery. The ideal operation for revision is still controversial and varies according to the type of the patient and cause of failure. So, malabsorptive surgery such as Roux en-Y gastric bypass is considered the procedure of choice in sweet eaters after failure of weight reduction following LAGB while LSG might be enough for bulk eaters.

Yoon and his colleagues ${ }^{8}$ considered LSG as a bridge procedure that may need a following operation.

Being of low learning curve and short operative time LSG evolved as a standalone bariatric surgery that not only acted as a restrictive procedure by reducing the size of the stomach but by having hormonal mechanism as well through reducing plasma level 
of ghrelin which regulates hunger.

The altered anatomy and massive scar tissue in revisional surgery increase morbidity and mortality rates. That's why inspite of the only short term follow up outcomes, LSG had been proven by many reports to be a good optional revisional surgery for failed LAGB. ${ }^{8}$

The performance of LSG along with band removal in one stage or with interval following band removal is still controversial between authors and surgeons ${ }^{8}$

Sagie and his colleagues ${ }^{9}$ showed that LSG could be done simultaneously with band removal in case of absence of serious infection which might be there resulting in increasing rates of leakage commonly at the esophagogastric junction or the original position of the band.

Delaying of LSG after band removal was better choice in presence of inflammation or infection.

We excluded patients with those co-morbidities in our study to allow successful band removal and LSG in same procedure.

Although cumulative failure and complication rate reached up to $30 \%$ after $L A B G$, there is no optimum procedure for patients who need revision surgery ${ }^{10}$

In our study, conversion of failed LAGB to sleeve gastrectomy was done in all cases with no leak or significant morbidity except for $6.7 \%$ who had postoperative bleeding and controlled by blood transfusion and $10 \%$ patients with infection at reservoir site who was managed by daily dressings and antibiotics. These results compared favourably with previous studies as Acholonu and his colleagues ${ }^{11}$ who had major complications reaching $13 \%$ of their patients after conversion of band failure to sleeve gastrectomy. Foletto and his colleagues ${ }^{12}$ reported in 2008 in a series of 57 patients who had revisional LSG for failed LAGB that leakage and mortality rate was $5 \%$ and $2 \%$ respectively.

Similarly, some authors noted a major morbidity rate of $6 \%$ and a conversion to open surgery in $4 \%$ of cases of revisional LSG. Unlike these studies, we managed to perform band removal and LSG simultaneously. Gagner and his colleagues ${ }^{13}$ found that there was no difference in morbidity incidence rate between waiting for six months after gastric band removal to do LSG and performing LSG in the same sitting.

The main target of our study was to perform band removal and simultaneous LSG in uncomplicated gastric banding patients who failed to have weight loss or regain weight.
This was achievable in all cases with no need for staged surgery or conversion to open in any case.

The issue raised by many authors that it is not helpful to convert a restrictive procedure to another and that gastric bypass is a better procedure for failed LAGB is respected because of absence of long-term outcomes for our study. However, choosing LSG depends on the relative technical simplicity of it as well as the low post-operative maintenance required following it. Also, the fact that LSG can be easily revised to another procedure in the future put to us a back door.

In our study $65 \%$ of our patients had reduction in percentage excess body weight requiring no further management till now. Our results are comparable to other studies that reported reduction only between $16 \%$ and $55 \%$ over a similar follow up period ${ }^{7}$

We knew that our study had some limitations as the small size of our cohort which abandoned us from giving strong conclusions from our data. In addition, there was no long term follow up of the patient to see the actual result of the LSG and whether the patients will need a second operation or not. However, this cohort study represented the power of practice in a referral bariatric center. Moreover, the prospective nature of our study, standardization of surgical technique and our strict follow-up protocol added considerable weight to the validity of our findings. Finally, we had shown that sleeve gastrectomy could be done simultaneously after band removal in patients with failed LAGB and with good short outcomes. Although this procedure might be applied to all patients with failed LAGB, the optimum peri-operative results were seen only in patients with weight regain or fail to lose weight after LAGB but we had no enough data for patients' who did this approach with band complications.

\section{Conclusion}

From our study, we concluded that Laparoscopic gastric band removal with conversion to sleeve gastrectomy in one operation is applicable for patients with failure of weight loss or regaining weight after adjustable gastric band. It can be done with low incidence of complications, and yet a weight loss results that is similar to primary sleeve gastrectomy.

\section{References}

1. Greenstein RJ, Rabner G, Taler Y: Bariatric surgery vs conventional dieting in the morbidly obese. Obesity Surg. 1994; 4: 16-23.

2. Suter M, Calmes JM, Paroz A, et al: A 10 years experience with laparoscopic gastric banding for morbid obesity: high long-term complication and failure rates. Obes Surg. 2006, 16 (7): 829- 
835.

3. Favretti F, Segato G, Ashton D, et al: Laparoscopic adjustable gastric bandingin 1,791 consecutive obese patients: 12-year results. Obes Surg. 2007; 17: 168-175.

4. Karamanakos SN, Vagenas K, Kalfarentzos F, et al: Weight loss, appetite suppression, and changes in fasting and postprandial ghrelin and peptide-YY levels after Roux-en-Y gastric bypass and sleeve gastrectomy: A prospective, double blind study. Ann Surg. 2008, 247:401-407.

5. Brethauer SA, Hammel JP, Schauer PR: Systematic review of sleeve gastrectomy as staging and primary bariatric procedure. Surg Obes Relat Dis. 2009, 5(4): 469-475.

6. Nguyen NT, Slone JA, Nguyen XM, et al: A prospective randomized trial of laparoscopic gastric bypass versus laparoscopic adjustable gastric banding for the treatment of morbid obesity: Outcomes, quality of life, and costs. Ann Surg. 2009, 250: 631- 641.

7. Buchwald $H$, Avidor $Y$, Braunwald $E$, et al: Bariatric surgery: A systematic review and metaanalysis. JAMA. 2004; 292(14): 1724-1737.

8. Yoon YC, Yong JK, Kyung YH: Simultaneous laparoscopic band removal and sleeve gastrectomy: Case report and review of literature. Journal of Minimally Invasive Surgery. 2012; 15(1): 23-25.

9. Abu-Abeid S, Zohar DB, Sagie B, Klausner $\mathrm{J}$ : Treatment of intra-gastric band migration following laparoscopic banding, safety and feasibility of simultaneous laparoscopic band removal and replacement. Obes Surg. 2005; 15: 849-852.

10. Catona A, Gossenberg M, La Manna A, et al: Laparoscopic gastric banding: preliminary series. Obes Surg. 2011; 3(2): 207-209.

11. Acholonu E, McBean E, Court I, Bellorin O, Szomstein S, Rosenthal RJ: Safety and short-term outcomes of laparoscopic sleeve gastrectomy as a revisional approach for failed laparoscopic adjustable gastric banding in the treatment of morbid obesity. Obes Surg. 2009; 19: $1612-1616$.

12. Foletto $M$, Bernante $P$, Busetto L: Laparoscopic gastric rebanding for slippage with pouch dilation: results on 29 consecutive patients. Obes Surg. 2008, 18(9): 1099-1103.

13. Gagner M, Milone L, Yung E: Causes of early mortality after laparoscopic adjustable gastric banding. J Am Coll of Surg. 2008; 206(4): 664669. 